\title{
Application and Research on The General Gateway for MODBUS Protocol and Siemens Insight Software
}

\author{
Haiyan Wang ${ }^{1,}$, Xiguang Liü,b \\ ${ }^{1}$ Zhejiang Zhejiang Police Vocational Academy Hangzhou City Zhejiang Province, China \\ ${ }^{2}$ Hangzhou Rayleigh acoustoelectric technology company Hangzhou City Zhejiang Province,China \\ ahz_why2002@163.com, bIxg@715.cn
}

Keywords: General Gateway, MODBUS, Insight, Siemens

Abstract. Modbus Protocol is commonly used in Building Automation System devices. Siemens compony with good quality and high quality services, is one of the largest supplier for Building Automation System in China, but Insight software of Siemens only provides OPC and BACNET Protocol for other brand building products (called third party device), the devices does not support the above two kinds of Protocols should be Converted to OPC Protocol to enter the data system of Insight. For Siemens does not provide the gateway conversion software, the paper around Modbus Protocol、OPC protocol and Insight software, designed the general gateway to connect the Modbus device into Siemens Insight software, in order to reduce dependence on a single supplier, improve productivity, reduce device idle, realize information sharing, improve the efficiency of the system, provide guarantee for the integration of intelligent building system.

\section{Introduction}

Building automation system (BAS) as an important control system of intelligent building, is the main management of many mechanical and electrical devices.

Currently, BAS devices from different manufacturers, communication interface and protocol have not been standardized. Porotools such as Modbus protocol, OPC, LONTALK protocol, BACNET protocol and M-BUS are commonly used. Such sui generis situation does not apply to the current demand of system integration in big data era, it becomes the bottleneck in the development of building construction, so designing a general gateway is the development trend.

According to the domestic situation, third party devices connected to the building automation system most use the Modbus protocol. Siemens as one of the largest domestic supplier,its Insight software only provides OPC and BACNET Protocol for the third party devices, other protocol devices must be converted to OPC to enter the data system of Insight software by a software gateway, Insight does not provided the gateway, that is generally written by the distributors or integrators .

But because the gateway development requires engineers having comprehensive knowledge, such as automation control, computer software, all kinds of communication protocol, etc, Domestic engaged in building automation engineers are few.

This paper designed a set of universal gateway software used for Siemens Insight software to connect Modbus devices, Software guides debugging personnel to complete the communication settings in the wizard way without programming requirements, the project cost and the unstable factors are reduced. The gateway software provides the basis for information sharing and makes the adjustment and linkage cross system possible.

\section{Function analysis}

A bridge between Modbus protocol devices and OPC devices, realized the data communication between different protocols. The gateway software needs to get the point value from Modbus devices. After data format conversion, the value will replace the matching OPC value label, or pass the value changes of OPC flags to the matching Modbus devices point.

General gateway software should have the following functions: 
(1) Providing the system configuration function to guide the system engineer to complete the system configuration.

It includes the name and location of OPC Sever, Serial number, Communication format, Device address, Bit address, Function code, Data format, etc.

(2) Support for multiple user permissions, distributing different system permissions to different personnel .

For example, engineers have the permissions to configure and modify the system, managers have operator account, enable or disable some devices or bit, The operator has the right to start or stop the gateway or quit the software. Some devices are in abnormal state for maintenance or temporary failure, managers can temporarily disable the devices, so the system can automatically stop access to the disable devices.

(3) Providing the human-computer interaction interface.

The interface can display the summarizing communication status, instantly outputs the exception information .It can also store the user's important operation and exception information in the log file for future inquiry.

(4) Intelligent analysis function.

The gateway software must deal with the abnormal situation of the system, avoiding unnecessary resource usage, such as when unable to connect the OPC Server, the access to the Modbus device should be stopped until the connection recovery. When a device is abnormal, the software can stop its continued access, automatically identify and mark, and timing detect that is normal or not, the original access pattern will recover till the device is normal.

(5) 4 basis points of Insight are supported.

The 4 basis points are analog input, digital input, analog output and digital output.

\section{Brief Protocol introduction and design ideas}

\section{Modbus Protocol}

The Protocol is a common language used in the electronic controller, it defines the message structure that the controller can understand and use, ignoring how they communicate through the network.

Modbus protocol including ASCII, RTU, TCP and so on, without specified physical layer. The Modbus protocol adopts the master and slave device working mode of "asking and answering", the master device of the inquiry is sent out, and the response is from the slave device.

\section{OPC Protocol}

OPC is based on OLE technology, providing OLE/COM standard interface for process control of industrial standard, composed of client and server with the client / server architecture. OPC specification provides two kinds of interface: the OPC Custom Interfaces and the OPC Automation interfaces. This paper used the OPC Automation interfaces.

\section{Design ideas}

The universal gateway software is suitable for different projects with integrated system configuration and real-time operation functions.

The gateway software is running on a unified database, including user management, system configuration, real-time communication, as shown in Fig.1.

\section{General gateway software design}

The general gateway software is composed of user management module, system configuration module, system management module, help module and instant communication module, as shown in Fig.2. 


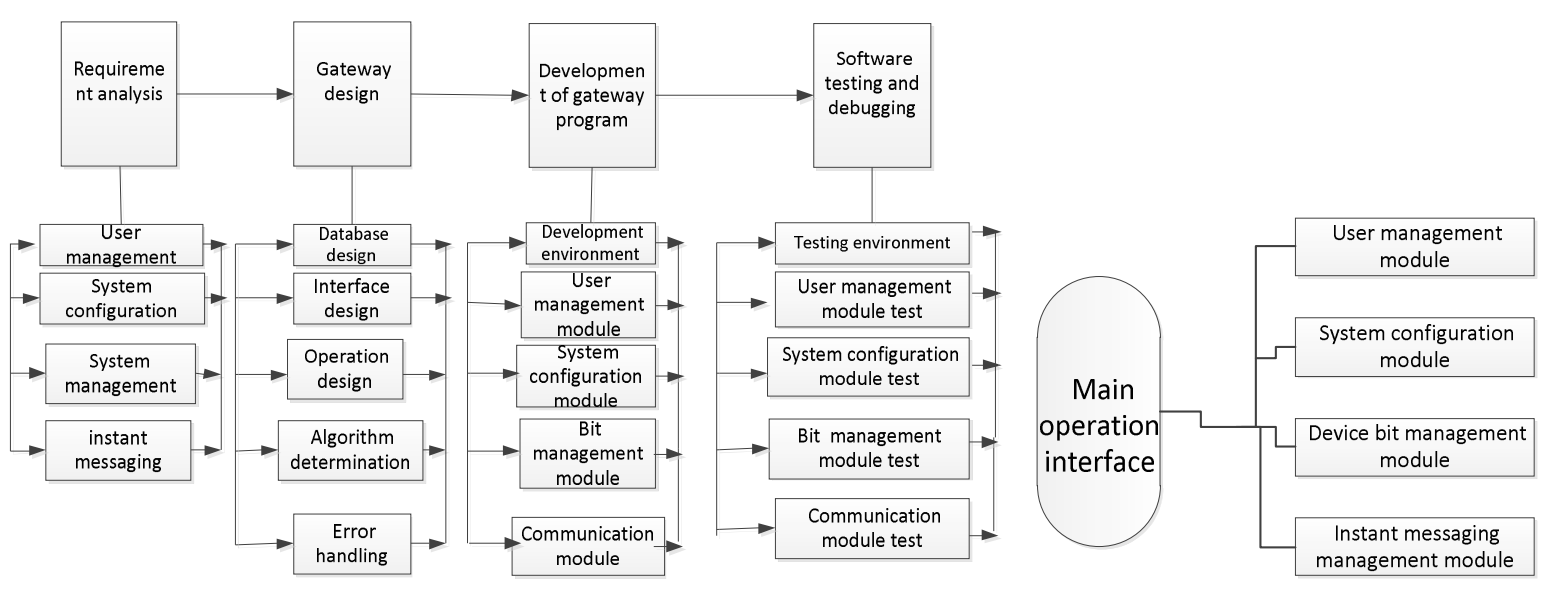

Fig.1 Gateway design flow

Fig.2 System structure

The user management module have two functions, they are user login management and account management ,mainly to completing the password modification, user cancellation, system exit, increase users, delete users, etc.

The system configuration module supports two kinds of configuration modes: manual and wizard, which mainly completes the inspection of the hardware connection, the connection of Server OPC, the configuration of serial port parameters, the bit configuration, and the direction of the third party device. $s$ name and address .

Device bit management module is to complete the bit disable and enable operation of the third party device.

Instant communication module is the core module of the gateway software, it can complete the communication with the device supported Modbus protocol, and for data processing, updating the Server OPC label, receiving change data from Server OPC and sending them to the Modbus device.

\section{User management module design}

The user management module is mainly shown in table 1.

Table 1 User management function table

\begin{tabular}{|c|c|c|}
\hline Sub module & Function & $\begin{array}{l}\text { Output } \\
\end{array}$ \\
\hline User login & Check with database records & $\begin{array}{l}\text { Global variable User, User ID, function } \\
\text { authorization }\end{array}$ \\
\hline $\begin{array}{l}\text { Add user } \\
\text { Delete user } \\
\text { Modifypassword }\end{array}$ & Manage user accounts & Modify staff table \\
\hline Cancellation & $\begin{array}{l}\text { Disable other functions except } \\
\text { login }\end{array}$ & \\
\hline Exit & Forced login and exit system & \\
\hline
\end{tabular}

The followed is to take the Login function as an example to illustrate its design and work flow, Fig. 3 is the work flow chart.

\section{System configuration module}

It provides interface and configuration guide for engineers to configure system configuration. Configuration guide uses the way STEP BY STEP to guide the system engineers to complete OPC Server, serial port, device and bit configuration. After configuration, the engineer can directly modify the system configuration through other sub modules provided in the module.

The system input is configured by the engineer, output is from the System configuration table for the database, Serial port configuration table, device table and bit table. System configuration table records the number of serial port, Serial port configuration table records the number of devices in the serial port, the Device table records the number of bits of the device. 


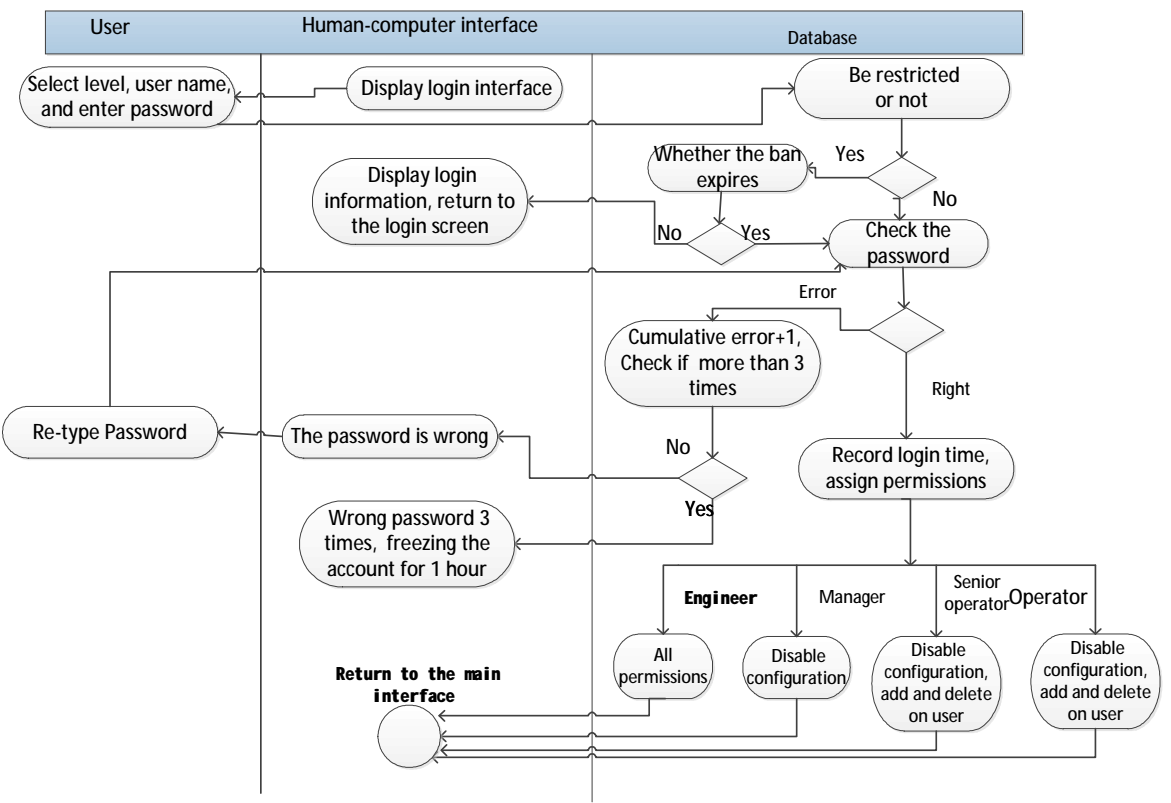

\section{Device bit management module}

Fig.3 User login flow chart

This module is mainly used by managers and senior operators to shield the long term not used of the serial port, device and point, or temporary shielding failure of device to improve the performance of the system. Therefore, the input of bit management module is from the manual input by the engineers, administrators and senior operators, the data format for the logic value is 0 and 1,0 means that the device is available, 1 means the device is disabled. The System output is from the management field content of the database serial port, device, the bit configuration table.

When a device fails, the system will automatically be shielded and regularly test if it returns to normal, before release the shielding, the system will skip over access and detection of the shielded bit.

This module includes three functions: serial port management, device management and bit management, as shown in table 2. The module's premise of execution is that the login personnel have the appropriate permissions, and the gateway is in a stop state.

Table 2 Function table of device bit module

\begin{tabular}{lll}
\hline $\begin{array}{l}\text { Sub module } \\
\begin{array}{l}\text { Serial port } \\
\text { management }\end{array}\end{array}$ & $\begin{array}{l}\text { Shielding some serial ports, at the same time, Modify the serial port management status } \\
\text { all the devices and the bits belong to it are } \\
\text { shielded }\end{array}$ \\
$\begin{array}{l}\text { Device } \\
\text { management }\end{array}$ & $\begin{array}{l}\text { Shielding some devices, at the same time, all } \\
\text { bits of the devices are shielded. }\end{array}$ & $\begin{array}{l}\text { Output } \\
\text { Modify the device management status in the } \\
\text { device table }\end{array}$ \\
$\begin{array}{l}\text { Bit } \\
\text { management }\end{array}$ & Shielding some bits & Modify bit management status in a bit table
\end{tabular}

Instant messaging module

This module is the core module of the software, data exchange between Server OPC and Modbus devices has been realized by reading the system configuration and the bit position table in the database.

This module uses three interrupt modes, that are communication interrupt, timer interrupt and data update interrupt, to control data acquisition, conversion and transmission . Instant messaging module structure is shown in Fig.4, the solid line in Fig.4 shows the direct call, the dotted line shows control module operation by setting the interrupt or sign . 


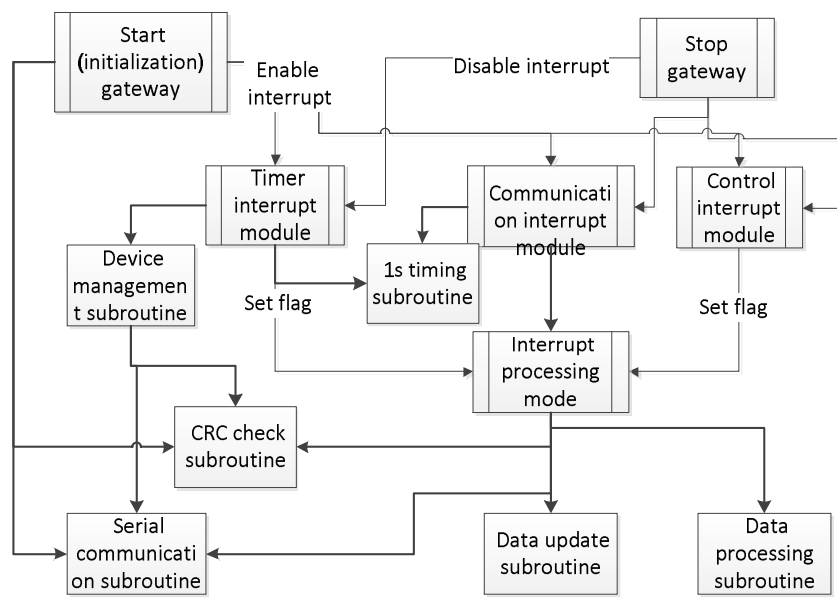

Fig.4 Instant messaging module structure

\section{Software debugging and testing}

\section{Test class}

According to GB/T15532-2008, Unit test and integration test are used.Unit test using a small piece of code written by the developer to test the function is correct or not, the function is very small but very clear . ${ }^{[6]}$

Integration test is based on Unit test, in accordance with the design requirements, assemble all modules into subsystems or systems.

\section{Test method}

Dynamic white box test is used to test the program, that is used the code to see the function and implementation of information to determine what needs to be tested, which do not test, how to carry out the test. ${ }^{[7]}$

\section{Platform for testing}

Operation system: windows 7 、Windows XP

Development Software: VB6.0

Run test software : Insight 3.8、OPCserver、SoftController

Tool software : COMMIX1.3, Virtual Serial Port Driver 6.0

Self-developed device simulation software

\section{The hardware for gateway development and testing}

One notebook PC, one desktop PC

One RS232 serial USB converter

One RS485-RS232 converter

Third party devices: Sevral multi parameter electric meters of Sifeier brand, transformer temperature controllers of Lide brand

\section{User management module test}

(1) Executing function test, a comprehensive test that is tested the function to add and delete users, modify passwords, rights management . All functions are reliable.

(2) Using White box dynamic test method to cover all procedures, code coverage rate is $100 \%$, the program can be properly implemented

(3) Stability test: The mothods of entering user name and password in a variety of characters, multiple error codes and abnormal exit are used to test the stability of the program.

(4) Destructive testing: Deleting the database to test if the system has an error message, or if the system can bypass the login.

After the above tes, reliability and stability of the module meet the design expectations. Test generated personnel table is shown in Table 3. 
Table 3 Test generated personnel table

\begin{tabular}{cccccccc}
\hline \hline \multirow{2}{*}{ Number } & $\begin{array}{c}\text { User } \\
\text { name }\end{array}$ & $\begin{array}{c}\text { Pass } \\
\text { word }\end{array}$ & Level & $\begin{array}{c}\text { Additional } \\
\text { power }\end{array}$ & creation date & Recent login & Restriction sign \\
\hline 1 & gcs & 1 & 4 & False & $2016 / 8 / 170: 26: 14$ & $2016 / 9 / 1920: 26: 56$ & False \\
2 & gly & 111 & 3 & False & $2016 / 8 / 171: 26: 03$ & $2016 / 9 / 1919: 57: 26$ & False \\
3 & gjc & 123 & 2 & False & $2016 / 8 / 171: 26: 45$ & $2016 / 9 / 1919: 57: 13$ & False \\
4 & czy & 123 & 1 & False & $2016 / 8 / 171: 26: 58$ & $2016 / 9 / 1920: 00: 28$ & True \\
5 & ggg & 111 & 2 & False & $2016 / 8 / 2119: 58: 12$ & & False \\
10 & ddd & 111 & 3 & False & $2016 / 9 / 1116: 15: 31$ & & False \\
11 & sss & 111 & 1 & False & $2016 / 9 / 1116: 57: 06$ & $2016 / 9 / 1919: 57: 05$ & False \\
12 & 1 & 1 & 1 & False & $2016 / 9 / 1117: 00: 16$ & & False \\
\hline
\end{tabular}

\section{System configuration module}

The module's input is manual, and the output of the system is recorded into the system configuration table, the serial port configuration table, the device table and the bit table.

This module mainly takes the interface operation and the table as the test result.

(1) First, according to the designed rout to test, the result is correct.

(2) In the view of customer, entering or randomly clicking the order of the control, A lot of mistakes have occurred. Mainly error is that the content is empty or the variable is not been setted.

Solutioln: Added fault tolerant code in the program. Part of the interface will disable the subsequent control or button, only the operation has been completed or has made the right choice, the disable things will appear or be effective.

(3) Fault tolerance test: Input inapporopriate content or the required content does not do input.

Solutioln: In the design of the database, as far as to carry out the fault-tolerant design ;for a fixed input, using the drop-down box or list box to select input; for the input must use keyboard, the code design input range verification functin.

\section{Device bit management module}

The function of the module is very simple, only need to select the appropriate device to execute disable or enable operation.

\section{Instant messaging module test}

The test is divided into two steps. The first step is to test the CRC calibration program and the data conversion program which affect the correctness of the program. The rest function module is put into the second step combination test. The second step is to use OPC as Server OPCJ.DADemoServer.1, using virtual serial port software and self written virtual device program to test the module by combination test

\section{(1) CRC test}

A comparative test between interface soft and communication assistant tool is carried out, the character is converted to 16 Decimal to facilitate comparison, the comparison is shown in Fig.5 .

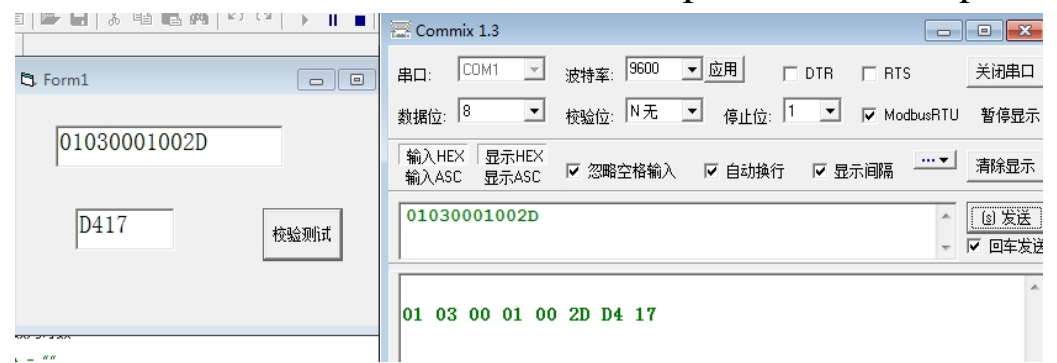

Fig.5 CRC16 program test comparison

\section{(2) Combination test}

The combination test is mainly to test the combined operation of program modules, and the data transfer accuracy been the test serial device and OPC Server.

Insight OPC Server is achieved by OPCJ.DADemoServer, who simulates serial connection through the virtual serial port software, Three group virtual serial ports has been established ,they are $1-5,2-6,4-7$. 


\section{(3) Running test}

Simulation test of the 6 points that is generated in the configuration process is carried out, The gateway program sends a read device instruction to a virtual device, after setting time, the virtual device program sends thev text box content $(55,66,77)$ of the "send value" tag . Use the OPC client tool to show the actual value of 6 points in the Server OPC, which is shown in Fig.6.

\begin{tabular}{|c|c|c|c|}
\hline \multicolumn{4}{|c|}{ 5. OPCJ.DADemoServer.1 - FactorySoft OPC Client } \\
\hline File & OPC View Help & & \\
\hline \multicolumn{4}{|l|}{ 8) } \\
\hline Tag & & Value & Time \\
\hline NA1 & & 55 & 09/20/11 04:18:03 \\
\hline NA2 & & 55 & 09/20/11 04:18:16 \\
\hline SA1 & & 0 & $09 / 20 / 1104: 18: 52$ \\
\hline NA3 & & 66 & 09/20/11 04:19:06 \\
\hline NR1 & & 66 & 09/20/11 04:20:54 \\
\hline NA5 & & 77 & $09 / 20 / 1104: 19: 15$ \\
\hline
\end{tabular}

Fig.6 OPC client tools display content

Through the above test, it is proved that the instant communication module realizes the design function.

\section{Conclusions}

The gateway is necessary for the Modbus and OPC communication protocols, using SIEMENS Server OPC and the gateway software to instead of the original hardware gateway, which is Estimated that $80 \%$ of the project can be replaced by the software, the project has a broad market value.

\section{Acknowledgement}

This work is supported by Public welfare project of Zhejiang provincial science and Technology Department of China (No. 2017C33166).

\section{References}

[1]Insight ${ }^{\circledR}$ OPC Server Getting Started Manual 571-167 Rev. 3, May, 2001

[2] JIANG Jin, MAO Liu- ping, DAI Yu- xin. Exploiture and Research of OPC Server Based on Modbus Protocol. Journal of Hunan Institute of Engineering, 2005,15(2):11-14.

[3]Zhang yousheng. System analyst course. Peking: Tsing Hua University Publishing House, 2010.2:27-80.

[4]Xiao song, wang yu ,caoyue.Software test. Peking: China Machine PRESS, 2006.4:120-350

[5]Lin yong, Zhang leiqiang. Visual Basic 6.0 User programming manual. Peking: The People's Posts and Telecommunications Press,2002(1):12-120.

[6]Wan yakan. C++ Language and object oriented programming. Peking: Tsing Hua University Publishing House, 1998(2):78-200.

[7] Xie shnyi,Yang qiang,Xu qingping. Address Space Mangement of OPC UA for Common Infoumation Modle.Automation of electric systems, 2016,7(14):115-121.

[8]Fu Kuan, Zhang Taojia, Liu Shengnan, Zheng Jianfeng, Shi Ning. Implementation Of Real-Time Database Client Based On OPC Standard. Information and communications. 2016(8):188-190.

[9] OPC Foundation. 78714-0524, Data Access CustomInterface Standard Specification 2. 05 [S]. Austin,Texas: OPC Foundation,2002.

[10]OPC Foundation. Data Access CustomInterface Specification 3.0 ,2003.

[11] GB/T 19582.1-2008. Industrial automation network specification based on Modbus protocol Part one: Modbus application protocol.

[12]GB/T 19582.2-2008. Industrial automation network specification based on Modbus protocol Part two: Modbus protocol implementation guide on the serial link. 